\title{
Exploring pedagogical culture for accessibility education in Computing Science
}

\author{
Sarah Lewthwaite \\ National Centre for Research Methods, \\ University of Southampton \\ Southampton, UK \\ +442380592623 \\ s.e.lewthwaite@soton.ac.uk
}

\author{
David Sloan \\ The Paciello Group (UK) \\ 20-22 Bedford Row \\ London, UK \\ dsloan@paciellogroup.com
}

\begin{abstract}
This paper identifies some of the challenges of teaching and learning accessibility through the lens of pedagogy (which deals with the theory and practice of education). We argue that accessibility education in computing science presents a set of unique and challenging characteristics for those engaged in accessibility capacity building. Significant moves are being made to embed accessibility within academic curricula and professional domains. However, through a qualitative thematic review of the accessibility pedagogic literature, we find that the field lacks the pedagogic culture necessary to support widespread excellence in teaching and learning. Nonetheless, our review identifies aspects of this small but important literature that indicate how a pedagogic culture for accessibility can be stimulated through research, debate and discussion, to promote a more pedagogically-grounded approach to the field as a whole.
\end{abstract}

\section{Categories and Subject Descriptors}

H.5.2 [User Interfaces - Evaluation/methodology]; K.3.2 [Computer Science and Information Science Education Computer science education] K.4.2 [Social Issues - Assistive technologies for persons with disabilities].

\section{General Terms}

Management, Documentation, Design, Human Factors, Theory.

\section{Keywords}

Web accessibility, education, pedagogy, disabled people, teaching and learning, social inclusion, guidelines, user experience.

\section{INTRODUCTION}

Business, legal and social motives for accessibility and inclusion mean there is a growing demand for professionals with the knowledge and skills to produce and procure digital resources that are accessible to people with disabilities. As a result, there is a need to improve the extent and quality of education in accessibility, in order to provide a supply of professionals with the necessary accessibility knowledge, research and leadership skills, beyond a basic coverage of guidelines and corresponding techniques [8]. An example of this demand is the Teach Access

\footnotetext{
Permission to make digital or hard copies of all or part of this work for personal or classroom use is granted without fee provided that copies are not made or distributed for profit or commercial advantage and that copies bear this notice and the full citation on the first page. Copyrights for components of this work owned by others than the author(s) must be honored. Abstracting with credit is permitted. To copy otherwise, or republish, to post on servers or to redistribute to lists, requires prior specific permission and/or a fee.

W4A 2016, April 11-13, 2016, Montreal, Canada.

Copyright is held by the owner/author(s). Publication rights licensed to

ACM. ACM 978-1-4503-4138-7/16/04 ...\$15.00
}

DOI: http://dx.doi.org/10.1145/2899475.2899490
Initiative (http://teachaccess.org), which was established in 2015 as a partnership between organizations in the technology industry, universities, and accessibility advocacy. It aims to advance accessibility in higher education curricula, to define the knowledge and skills required by industry that should be covered in education programs, and to demonstrate the employment opportunities for graduates with solid knowledge of accessibility.

Defining the required accessibility knowledge and skills helps with the task of integrating accessibility into a general Computing Science or related undergraduate curricula. However, as courses and qualifications develop, there is also a pressing need to develop the pedagogies - the learning theories and teaching approaches-appropriate to accessibility, so that the education process is effective. To this end, we argue that greater attention should be given to the development of the 'pedagogic cultures' that educational research shows are necessary to support excellence in teaching and learning (see [13], [27]).

\section{PEDAGOGY AND ACCESSIBILITY}

To understand the pedagogic challenges relating to accessibility education, we need to consider the extent of knowledge and skills that accessibility requires, and thus the pedagogic approaches best suited to teaching the subject. In this paper, we do not seek a unified definition of accessibility, but for the purpose of clarity, we take this to cover all aspects of the process of creation of web sites, apps, software and other digital products that can be successfully used by people with disabilities.

\subsection{Pedagogical Content}

It is our view that accessibility requires a unique combination of theoretical understanding (drawing upon multiple disciplines), procedural knowledge and technical skills competence. A thorough understanding of accessibility covers an array of topics that engage with the complexity of accessibility as a sociotechnical challenge, and the knowledge and skills to create digital resources that are optimally accessible. Accessibility pedagogy must transmit both tested approaches as well as an inclination to question these approaches ruthlessly.

Accessibility draws heavily from computing science, focusing on the interplay between operating systems, assistive technologies, browsers and other applications and digital content, and the technical process of creating device-independent, multimodal, flexible interaction. It also draws from human-computer interaction, taking aspects of ergonomics and psychology to understand human characteristics and behavior, and disability studies, especially the factors that influence discrimination against people with disabilities, and how discriminatory activity by individuals and organizations can be addressed. Applying 
accessibility knowledge in the context of an organization's activities and structure is also part of the educational challengeunderstanding how responsibility for aspects of accessibility can be most effectively shared in standard industry digital product development and publishing processes.

However, literature suggests that some learner characteristics represent a significant hurdle for those teaching accessibility. For example, Edwards et al. report that Computing Science students consider HCI 'easy' and somehow commonsense [5]. There is also a challenge of developing empathy, especially amongst young cognitively and physically high-performing students, for the nature and the impact of accessibility issues experienced by people with sensory, cognitive and motor disabilities (see [16], [19]).

Some approaches to accessibility education beyond traditional lab and classroom-based teaching have been applied. One example is hackathons, where representatives from disability organizations and/or people with disabilities have been directly involved along with students in user research, requirements establishment and evaluation stages of development projects.

\subsection{The characteristics of accessibility learning and teaching}

\subsubsection{The pedagogic literature}

To explore the nature of emerging, research-informed pedagogical content knowledge (PCK) [23] in the teaching of accessibility, we undertook a review of publications from 2005-2015, modelled on Kilburn et al's investigations into pedagogical culture in advanced research methods teaching [13]. PCK refers to the intersection between a teacher's general pedagogical know-how-how to teach - and the content that is particular to a discipline - what to teach. It bridges disciplinary and pedagogic expertise.

To identify all peer-reviewed outputs on the learning and teaching of accessibility, the Web of Science bibliographic database was used for its deep coverage of computer and allied sciences. A high sensitivity search was conducted on the terms: accessibility AND (teaching OR learning OR education OR training OR instruction OR "professional development"). Over 2500 titles were returned and hand-searched to identify those that focused on the teaching of accessibility specifically. This discounted, for example, papers dealing with the development of accessible materials, systems or websites for disabled students. References and lists of received citations were then checked to discover new publications. From this point we checked papers for substantive pedagogic content and focus on teaching practices, and for discipline-focused on the teaching of HCI and software/web design and development. This process resulted in a shortlist of 23 available papers, three introductions to thematic sessions, two posters and one $\mathrm{PhD}$ thesis ${ }^{1}$.

\subsubsection{Current pedagogies?}

The available literature was characterized by several meta-themes. Notably, the literature is a small one and, within this, crosscitation was uneven. This is a cause for concern because, for pedagogic culture to thrive, ideas must be shared, debated, empirically examined, and developed [25]. At present, the literature appears under-developed and fragmented. We also found that the majority of pedagogic research papers comprise of

\footnotetext{
${ }^{1}$ The full reference list is available via: http://eprints.soton.ac.uk/388799/
}

teachers' reflections on their own practice and course design (e.g. [12], [26]). This accords with Putnam et al., who observed the pedagogic literature is often characterized by 'first person reflections about teaching accessibility' [20]. Reflective case studies of pedagogical practice and development have value for establishing knowledge in a field and collectively such work can contribute to the much needed task of building pedagogic culture [18]. However, there is a need for the field to move beyond accounts of specific modules and teaching teams so educators can call upon a substantive body of literature characterized by systematic debate, cross-case investigation and evaluation of teaching and learning to inform their practice. Notable exceptions include: a substantial comparative study concerning the teaching of accessibility at three universities in the USA and Europe [3] and research that raises the level of investigation to a communitylevel, through interviews with 18 accessibility instructors across 15 institutions [20].

The contexts and details of the courses being taught vary across the literature. However, similar challenges and pedagogical themes emerge, including: the lack of a pedagogical culture to support accessibility teaching and learning [20]; active pedagogies focused on project-based and problem-based learning ([1], [10]) the promotion of tool-based approaches to answer perceived learning needs, with attendant discussions of learning by doing, immersion, and simulation (e.g. [2], [7]); embedding accessibility within a wider HCI curriculum (e.g. [26]); approaches that are understood to facilitate student empathy (e.g. [20], [11]), and engaging people with disabilities for the purposes of service- and collaborative learning (e.g. [21]).

Notably, levels of engagement with pedagogy and theory, particularly concerning disability, vary widely. Nonetheless, there are reasons for optimism. Novel research exploring typologies of motivation [22] and expanding pedagogic understanding by drawing on neighboring disciplines [9] show that there are many rich seams of pedagogical research available for exploration. Introductions to special thematic sessions and position papers gesture to an appetite for dialogue and peer-to-peer learning amongst educators. However, records and outputs are not visible to wider audiences.

\subsection{Challenges to the field}

So, given the emerging themes from the literature review, what challenges do we face in building a pedagogical culture for accessibility?

\subsubsection{Changing curriculum influences pedagogy}

Within accessibility there is no formally agreed curriculum [4]. Accessibility expertise is fragmented and distributed. As an academic topic, accessibility struggles for visibility, typically categorized as a sub-group of HCI, and sometimes of web development. This is reflected in the lack of coverage in literature targeted at supporting teachers in providing effective accessibility education. Working in this fast-changing environment requires constant vigilance and skills development on the part of both learners and teachers. Yet, while technology and development methods evolve, the accessibility maturity of many organizations that produce digital content, tools and services is such that accessibility activity is most commonly presented as evaluation and repair of existing resources, rather than the application of a comprehensive inclusive design strategy that keeps pace with innovation [16].

Accessibility expertise is also characterized by the development of new knowledge. The research that generates this knowledge 
arguably differs from other kinds of HCI research, as it requires a critical understanding of disability, not only in terms of being sensitized to, and accommodating of, diverse human capability but also in terms of recognizing the identity politics of disability and critically engaging with the ways in which technologies create 'disabled' experiences by excluding certain forms of use and engagement.

\subsubsection{The problem of 'best practice'}

Guidelines, most notably the Web Content Accessibility Guidelines, published by $\mathrm{W} 3 \mathrm{C}$, have been influential in enhancing web-development practices for the benefit of disabled people. However, as the de-facto source of education about accessibility, guidelines and their associated learning materials need to part of a wider learning ecology. Current discourse is characterized by what Stacey [24] describes as the high-agreement, high-certainty territory of standards, guidance and monitoring of best practicewhich might also include accessibility analytics. These alone can undermine the development of pedagogic culture by prescribing limited pedagogic practices [17].

As learning tools, standards and other 'best-practice', 'what works' approaches are problematic as they implicitly ignore the sociocultural aspects of learning, namely the interactions between instructors and learners of accessibility and their different cultures and identities. While common in the framing of education and technology, these approaches suggests that education is something that is done by an educator to a learner. This ignores the diversity of learners engaged in our accessibility community, their values, situations, aims, pre-existing knowledge and learning journey. In this respect, many of the learning resources available to accessibility learners are presented with one-size-fits-all content which is antithetical to the inclusive practices which the accessibility community seeks to promote.

An additional practical challenge is the catch-up role that accessibility frequently plays in the fast-paced technology sector. Emerging technologies and development practices become popular before accessibility issues have been fully addressed, and these issues are encountered by developers or assistive technologists who then start the process of trying to establish reliable workarounds while advocating for improved accessibility support in the technology or practices in question. The challenge of keeping current with this dynamic situation in accessibility education and its effect on what is considered 'best practice' also needs to be considered.

\subsubsection{Pedagogic culture}

In accessibility, we observe a lack of pedagogic space, and a lack of 'pedagogic culture' [26]; that is a lack of debate, investigation and evaluation regarding how accessibility is taught and learned. Our review of the literature shows that insights in this field tend to be based in individual accounts more than detailed pedagogic research. We find a developing research base. However, the limitations of this small literature suggest that it contributes little to how students, professionals and practitioners actually learn accessibility, and little for instructors to use in developing their own practice. Moreover, we observe that, while there are encouraging aspects of pedagogical scholarship, there remains little evidence of the systematic debate, in terms of cross-citations within the literature, or substantial dialogue between academic and practice contexts that signal pedagogic culture [25].

\subsubsection{Capacity building}

Given the current context, in which significant resources are being directed towards expanding professional development and embedding accessibility within academic programs, a number of key questions emerge. How do web developers and other professionals engaged in the shared project of accessibility most effectively learn about accessibility and gain the skills, knowledge and understanding required for their tailored application in the real world? How can accessibility curricula be developed in a way that is current, relevant, and strikes the right balance of computing and social science topics necessary for a holistic understanding of accessibility and its challenges? To what extent should accessibility be given the status of a stand-alone, mature component of a computing, digital design or even HCI curriculum, and to what extent should it be integrated into relevant parts of the existing curriculum such that accessibility is seen as a core quality of all stages of the design and development lifecycle? And, given all the above, how do instructors of accessibility most effectively develop and use their pedagogical knowledge for developing the learning of others?

\section{BUILDING PEDAGOGICAL CULTURE}

This paper describes some of the unique pedagogic challenges that accessibility presents to teachers, instructors and others developing accessibility teaching and learning. We identify a lack of pedagogic culture and the unique conditions within accessibility discourse that can be seen to perpetuate this. This follows from observations previously made [27]. However, this literature does suggest ways in which pedagogic culture might be developed. To promote this development, we apply concepts from educational research into pedagogical culture ([13], [17]) to offer the following four strategies as potential ways forward.

\subsubsection{Engaging with, and sharing pedagogy}

At present, there is a need to deepen the conceptual and theoretical understanding that is peculiar to accessibility as a discipline. There is a need to make pedagogy explicit and to undertake research to this end. There is significant scope for accessibility instructors to engage with wider literatures on pedagogy, work exemplified by Kane's engagement with pedagogies from architecture and industrial design [9]. In addition to the literatures of neighboring disciplines such as Information Sciences and rehabilitation disciplines, we encourage interdisciplinary moves towards the social sciences, in particular, the substantial and deep literature available in inclusive education, inclusive research methods, and the burgeoning literature within disability studies.

\subsubsection{Moving to community-level discussion}

In this paper we have sought to expand the lens of interest beyond individual accounts of 'what works' to a community-level, considering diverse sources of knowledge from and about accessibility instruction. By doing so, we hope to spur dialogue, by introducing pedagogic literature and concepts that may be unfamiliar to accessibility experts, and encouraging named and reflexive engagement with the pedagogies that professionals and instructors already use. At present, workshops, networks, journals and other forums dedicated to fostering accessibility pedagogy lack visibility. Thematic sessions focused on pedagogy and accessibility in high profile conferences, particularly at ICCHP (e.g. [4],[27]), suggests that there is an appetite for these. These and other forums should be encouraged, as should generous work to build the discipline through shared curricula and curricular development (as seen in [15], [28]). 


\subsubsection{Promoting extended professionalism}

Fostering dialogue in teaching, and research into the teaching of accessibility allow for 'taken-for-granted' frames of reference to be scrutinized [14] with participants in this work being potentially changed by it [17]. Nind et al. further argue that this engagement can be a catalyst for extending professionalism - with professional instructors extending beyond knowledge as technical expertise to embrace a reflexive, strategic, critical or problematizing approach to their teaching and to their research and development work [17]. This suggests clear benefits for accessibility education as a whole, beyond academia.

\subsubsection{Creating research/teaching spaces}

There is a need to widen and deepen pedagogy development. Hasty moves towards perceived solutions can limit dialogue. Creating inclusive research/teaching spaces that foster 'radical collegiality' [6] may enable more transformative collaboration to expose and develop the pedagogical content knowledge [23] of accessibility. While it has been beyond the scope of this paper to engage with accessibility blogs, course materials and other literatures, there is clear value in drawing this into scholarship (as seen in [3], [22]) and opening scholarship - via open access publishing and other channels - to professional audiences.

\section{REFERENCES:}

[1] Alonso, F., Fuertes, J. L., Gonzalez, A. L. \& Martinez, L. 2010. Using Collaborative Learning to Teach WCAG 2.0. ICCHP '10, Lect Notes Comput Sc, 6179: 400-403.

[2] Benavidez, C., Fuertes, J. L., Gutierrez, E. \& Martinez, L. 2006. "Teaching web accessibility with "Contramano" and hera." ICCHP '06, Lect Notes Comput Sc. 4061: 341-348.

[3] Bohman, P. R. 2012. Teaching Accessibility and Design-ForAll in the Information and Communication Technology Curriculum: Three Case Studies of Universities in the United States, England, and Austria, Utah State University. Thesis.

[4] Craven, J. \& Klaus, J. 2008, Accessibility: Education for web design and e-learning introduction to special thematic session. ICCHP '08, Lect Notes Comput Sc, 5195: 178-181.

[5] Edwards, A., Wright, P., \& Petrie, H. 2006. HCI education: We are failing - why?. In Proceedings of HCI Educators Workshop, Limerick, Ireland, March 23-24, 2006.

[6] Fielding, M. 1999. Radical Collegiality: Affirming Teaching as an Inclusive Professional Practice, Aust Educ Researcher, 26(2):1-34

[7] Freire, A. P., Fortes, R. P. M., Paiva, D. M. B., \& Turine, M. A. S. 2007. Using Screen Readers to Reinforce Web Accessibility Education. SIGSCE Bulletin 39(3): 82-86.

[8] Hengstberger, B., Miesenberger, K., Batusic, M., Chelbat, N. \& Garcia, A. R. 2008. Joint study programme on accessible web design. ICCHP '08, Lect Notes Comput Sc, 5105: 182198.

[9] Kane, S. K. 2007. Engaging Student Programmers as Inclusive Designers. SIGSCE Bulletin, 39 (3): 342-342.

[10] Katsanos, C., Tselios, N., Tsakoumis, A. \& Avouris N. 2012. Learning about Web accessibility: a project based toolmediated approach. Edu \& Info Tech, 17(1): 79-94.
[11] Kelle, S., Henka, A. \& Zimmerman, G. 2015. A personabased extension for massive open online courses in accessible design. Procedia Manufacturing. 3: 3663-3668.

[12] Keates, S. 2015. A pedagogical example of teaching Universal Access. UAIS, 14 (1): 97-110.

[13] Kilburn, D., Nind, M. \& Wiles, R. 2014. Learning as Researchers and Teachers: The Development of a Pedagogical Culture for Social Science Research Methods?, Br J Educ Stud, 62(2): 191-207

[14] Mezirow, J. 2003. Transformative learning as discourse. $J$ of Transformative Education, 1(1): 58-63.

[15] Miesenberger, K., \& Ortner, D. 2006. Raising the expertise of web designers through training - the experiences of BFWD - accessible web design (Barrierfreies Webdesign) in Austria. ICCHP '06, Lect Notes Comput Sc, 4061: 253-257.

[16] Newell, A. 2011. Design and the digital divide: insights from 40 years in computer support for older and disabled people. Morgan and Claypool Publishers.

[17] Nind, M., Kilburn, D. \& Wiles, R. 2014. Fostering discursive pedagogic spaces in capacity building. European Conference of Educational Research. Porto, Portugal, 2-5 Sept, 2014.

[18] Nind, M., Kilburn, D. \& Luff, R. 2015. The teaching and learning of social research methods: developments in pedagogical knowledge. Int J Soc Res Methodol 18(5) 455462.

[19] Poor, G. M., Leventhal, L. M., Barnes, J., \& Hutchings, D. R. 2009. Accessibility: understanding attitudes of CS students. ASSETS '09, October 25-28. Pittsburgh, USA.

[20] Putnam, C., Dahman, M., Rose, E., Cheng, J. \& Bradford. G. 2015. Teaching Accessibility, Learning Empathy. ASSETS '15, Lisbon, Portugal. ACM: New York.

[21] Rosmaita, B. J. 2007. Making service learning accessible to computer scientists. SIGSCE Bulletin, 39(1): 541-545.

[22] Rughinis, C. \& Rughinis, R. 2014. 'In my shoes' interaction sandbox for a quest of accessible design: teaching sighted students accessible design for blind people. UAHCI, Lect Notes Comput Sc, 8513, 64-74.

[23] Shulman, L. 1986. Those who understand: Knowledge growth in teaching. Educ Res, 15, 4-14.

[24] Stacey, R. 2012. Tools and techniques of leadership and management: Meeting the challenge of complexity. Abingdon: Routledge.

[25] Wagner, C., M. Garner, \& Kawulich, B. 2011. The state of the art of teaching research methods in the social sciences: towards a pedagogical culture. Stud High Educ 36(1): 75-88.

[26] Waller, A., Hanson, V. L. \& D. Sloan, D. 2009. Including Accessibility Within and Beyond Undergraduate Computing Courses. ASSETS '09, October 25-28. Pittsburg, USA.

[27] Weber, G. \& Abascal, J. 2006. People with disabilities: Materials for teaching accessibility and design for all. ICCHP '06, Lect Notes Comput Sc. 4061: 337-340.

[28] Whitney, G., Keith, S., \& Schmidt-Belz, B. 2010. The challenge of mainstreaming ICT design for all. ICCHP '10, Lect Notes Comput Sc. 6179: 583-59. 